\title{
Tropical resultants for curves and stable intersection
}

Luis Felipe Tabera

\begin{abstract}
We introduce the notion of resultant of two planar curves in the tropical geometry framework. We prove that the tropicalization of the algebraic resultant can be used to compute the stable intersection of two tropical plane curves. It is shown that, for two generic preimages of the curves to an algebraic framework, their intersection projects exactly onto the stable intersection of the curves. It is also given sufficient conditions for such a generality in terms of the residual coefficients of the algebraic coefficients of defining equations of the curves.
\end{abstract}

\section{Introduction}

In the context of tropical geometry, it is well known that two tropical curves may share an infinite number of intersection points without sharing a common component. This problem is avoided with the notion of stable intersection, [9]. Given two curves, there is a well defined set of intersection points that varies continuously under perturbations of the curves. This stable intersection has very nice properties. For example, it verifies a tropical version of Bernstein-Koushnirenko Theorem (cf. [9]). An alternative way of defining a finite intersection set is the following: given two tropical curves $f$ and $g$, take two algebraic curves $\widetilde{f}$ and $\widetilde{g}$ projecting onto the tropical curves. Then, the intersection of the two algebraic curves $\widetilde{f} \cap \widetilde{g}$ will project into the intersection of the tropical curves, $T(\widetilde{f} \cap \widetilde{g}) \subseteq f \cap g$. In general, the set $T(\widetilde{f} \cap \widetilde{g})$ depends on the election of the curves $\widetilde{f}$ and $\widetilde{g}$. We are proving that, if the coefficients of $\widetilde{f}, \widetilde{g}$ are generic, then the algebraic intersection will project exactly onto the stable intersection and there is a correspondence among

2000 Mathematics Subject Classification: 14M25, 14H50, 52B20.

Keywords: Tropical geometry, resultants, plane curves. 
the multiplicities of the intersection points. Moreover, given two curves, we compute residually dense sufficient conditions defining these genericity conditions. The method works in any characteristic and it is essential in the generalization of the geometric construction method of [13] to the non linear case. This also provides a particular case of Bernstein-Koushnirenko theorem for fields of positive characteristic. In order to prove this relationship, we introduce the notion of tropical resultant, as the tropicalization of the algebraic resultant.

The paper is structured as follows. In Section 2, we give a brief description of the algebraic context we will work in. Next, in Section 3 we recall the notion of stable intersection for plane curves and provide a brief discussion about its properties. Then, it is introduced the notion of tropical resultant for univariate polynomials (Section 4) and plane curves (Section 5). In Section 6 , we relate the stable intersection of tropical curves with the resultant of the curves and the generic preimage under the tropicalization map. We will provide conditions for the lifts (preimages) to be compatible with the stable intersection and the correspondence of the multiplicities. Finally, in Section 7 we present some comments and remarks about the results.

\section{Some basic notions in Tropical Geometry}

The algebraic context where the theory is developed is the following:

Let $\mathbb{K}$ be an algebraically closed field provided with a non trivial rank one valuation $v: \mathbb{K}^{*} \rightarrow \Gamma$. Without loss of generality, we may suppose that $\mathbb{Q} \subseteq \Gamma \subseteq \mathbb{R}$ and that $v$ is onto $\Gamma$. We denote by $k$ the residual field of $\mathbb{K}$ under the valuation. We will distinguish two main cases along the paper: the case whether $\operatorname{char}(k)=0$ (hence $\operatorname{char}(\mathbb{K})=0$ ) and the case wether $\operatorname{char}(k)=p>0$. In this case, either $\operatorname{char}(\mathbb{K})=p$ (equicharacteristic $p$ ) or $\operatorname{char}(\mathbb{K})=0$ ( $p$-adic case). It is also assumed that we have fixed a multiplicative subgroup $\Gamma^{\prime} \subseteq \mathbb{K}^{*}$ that is isomorphic to $\Gamma$ by the valuation map. The element $t^{\gamma}$ represents the element of $\Gamma^{\prime}$ whose valuation is $\gamma$. Any element $x$ of $\mathbb{K}^{*}$ can be uniquely written as $x=x_{0} t^{\gamma}$, where $v\left(x_{0}\right)=0$. We will denote the principal coefficient of an element $x$ of $\mathbb{K}$ by $\operatorname{Pc}(x)=\bar{x}_{0} \in k^{*}$ and $\operatorname{Pc}(0)=0$. We denote the principal term of an element by $\operatorname{Pt}(x)=\bar{x}_{0} t^{\gamma}$. The principal term of an element is only a notation, it is not, in general, an element of $\mathbb{K}$. If $y$ is an element of $\mathbb{K}^{*}, \operatorname{Pt}(x)=\operatorname{Pt}(y)$ if and only if $v(x)=v(y)<v(x-y)$.

The tropicalization map is minus the valuation, $T(x)=-v(x)$. The tropical semiring $\mathbb{T}$ is the group $\Gamma$ with the operations of tropical addition $" a+b "=\max \{a, b\}$ and tropical product " $a b "=a+b$. With these operations, $T(a b)=$ "T(a)T(b)" and, if $v(a) \neq v(b)$ or $v(a)=v(b)=v(a+b)$ then 
$T(a+b)=" T(a)+T(b) "$. Let $f=" \sum_{i \in I} a_{i} x "=\max _{i \in I}\left\{a_{i}+i x\right\} \in$ $\mathbb{T}\left[x_{1}, \ldots, x_{n}\right]$ be a polynomial of support $I$, where $x=x_{1}, \ldots, x_{n}, i=$ $i_{1}, \ldots, i_{n}, i x=i_{1} x_{1}+\ldots+i_{n} x_{n}$. The set $\mathcal{T}(f)$ of zeroes of $f$ is the set of points in $\mathbb{T}^{n}$ such that the maximum of the piecewise affine function $\max _{i \in I}\left\{a_{i}+i x\right\}$ is attained for at least two different indices. It is known (Kapranov's Theorem, [3]) that if $\widetilde{f}=\sum_{i \in I} \widetilde{a}_{i} x^{i}$ is any polynomial in $\mathbb{K}\left[x_{1}, \ldots, x_{n}\right]$ such that $T\left(\widetilde{a}_{i}\right)=a_{i}$, then $T\left(\{\widetilde{f}(x)=0\} \cap\left(\mathbb{K}^{*}\right)^{n}\right)$ is exactly the set of zeroes of $f$. Moreover, if $q \in \mathbb{T}^{n}$ is a point, let $J \subseteq I$ be the set of indices where the value $f(q)$ is attained and let $\alpha_{i}=\operatorname{Pc}\left(\widetilde{a}_{i}\right)$. We define the residual polynomial of $\tilde{f}$ over $q$ as:

$$
\widetilde{f}_{q}\left(x_{1}, \ldots, x_{n}\right)=\sum_{i \in J} \alpha_{i} x^{i}=\operatorname{Pc}\left(\widetilde{f}\left(x_{1} t^{-q_{1}}, \ldots, x_{n} t^{-q_{n}}\right)\right) \in k\left[x_{1}, \ldots, x_{n}\right]
$$

Then, it happens that:

Theorem 1. Let $\tilde{f} \in \mathbb{K}\left[x_{1}, \ldots, x_{n}\right]$ and $\left(\widetilde{b}_{1}, \ldots, \widetilde{b}_{n}\right) \in\left(\mathbb{K}^{*}\right)^{n}$ be any point, then there is a root $\left(\widetilde{c}_{1}, \ldots, \widetilde{c}_{n}\right)$ of $\widetilde{f}$ such that $\operatorname{Pt}\left(\widetilde{c}_{i}\right)=\operatorname{Pt}\left(\widetilde{b}_{i}\right), 1 \leq i \leq n$, if and only if $b=\left(T\left(\widetilde{b}_{1}\right), \ldots, T\left(\widetilde{b}_{n}\right)\right)$ is a zero of the tropical polynomial $f$ and $\left(\operatorname{Pc}\left(\widetilde{b}_{1}\right), \ldots, P c\left(\widetilde{b}_{n}\right)\right)$ is a root of $\widetilde{f}_{b}$ in $\left(k^{*}\right)^{n}$.

For a constructive proof of this theorem we refer to [14] or [6].

Let $C$ be a tropical plane curve defined as the zero set of a tropical polynomial $f=" \sum_{(i, j) \in I} a_{(i, j)} x^{i} y^{j}$ ". If we multiply $f$ by a monomial, the curve it defines stays invariant. We define the support of $C$ as the support $I$ of $f$ modulo a translation of an integer vector in $\mathbb{Z}^{2}$. Analogously, given an algebraic curve $C$ in $\left(\mathbb{K}^{*}\right)^{2}$ defined by an algebraic polynomial $\widetilde{f}=\sum_{(i, j) \in I} \widetilde{a}_{(i, j)} x^{i} y^{j}$, multiplying by a monomial does not change the set of zeroes in the algebraic torus $\left(\mathbb{K}^{*}\right)^{2}$, we also define the support of $C$ as the set $I$ modulo translations by an integer vector. If $C$ is a tropical curve, it may happen that there are polynomials with different support defining $C$, even under the identification by translations we have defined. Hence, when we define a tropical curve, we will always fix the support of a defining polynomial.

Let $I$ be the support of a tropical polynomial $f$, the convex hull $\Delta=\Delta(I)$ of $I$ in $\mathbb{R}^{n}$ is the Newton polytope of $f$. This object is strongly connected with the set of zeroes of $f$. Every tropical polynomial $f$ defines a regular subdivision of its Newton polytope $\Delta$. The topological closure of $\mathcal{T}(f)$ in $\mathbb{R}^{n}$ has naturally a structure of piecewise affine polyhedral complex. This complex is dual to the subdivision induced to $\Delta$. To achieve this duality we have first to define the subdivision of $\Delta$. 
Let $\Delta^{\prime}$ be the convex hull of the set $\left\{(i, t) \mid i \in I, t \leq a_{i}\right\} \subseteq \mathbb{R}^{n+1}$. The upper convex hull of $\Delta^{\prime}$, that is, the set of boundary maximal cells whose outgoing normal vector has its last coordinate positive, projects onto $\Delta$ by deleting the last coordinate. This projection defines the regular subdivision of $\Delta$ associated to $f$ (See [8] for the details).

Proposition 2. The subdivision of $\Delta$ associated to $f$ is dual to the set of zeroes of $f$. There is a bijection between the cells of Subdiv $(\Delta)$ and the cells of $\mathcal{T}(f)$ such that:

- Every k-dimensional cell $\Lambda$ of $\Delta$ corresponds to a cell $V^{\Lambda}$ of $\mathcal{T}(f)$ of dimension $n-k$ such that the affine linear space generated by $V^{\Lambda}$ is orthogonal to $\Lambda$. (In the case where $k=0$, the corresponding dual cell is a connected component of $\left.\mathbb{R}^{n} \backslash \overline{\mathcal{T}(f)}\right)$

- If $\Lambda_{1} \neq \Lambda_{2}$, then $V^{\Lambda_{1}} \cap V^{\Lambda_{2}}=\emptyset$

- If $\Lambda_{1} \subset \bar{\Lambda}_{2}$, then $V^{\Lambda_{2}} \subset \overline{V^{\Lambda_{1}}}$

- $\mathcal{T}(f)=\bigcup_{0 \neq \operatorname{dim}(\Lambda)} V^{\Lambda}$ where the union is disjoint.

- $V^{\Lambda}$ is not bounded if and only if $\Lambda \subseteq \partial \Delta$.

From this, we deduce that, given a fixed support $I$, there are finitely many combinatorial types of tropical curves with support $I$. These different types are in bijection with the different regular subdivisions of $\Delta$.

Finally, let $C$ be a tropical planar curve of support $I$ and Newton polygon $\Delta$, let $\Lambda$ be a one-dimensional cell of the subdivision of $\Delta$ dual to $C$, then, the weight of the dual cell $V^{\Lambda}$ is defined as $\#\left(\bar{\Lambda} \cap \mathbb{Z}^{2}\right)-1$, the integer length of the segment $\Lambda$.

\section{The notion of stable intersection}

One of the first problems encountered in tropical geometry is that the projective geometry intuition is no longer valid. If we define a tropical line as the set of zeroes of an affine polynomial " $a x+b y+c$ ", then two different lines always intersect in at least one point. The problem is that sometimes they intersect in more than one point. The usual answer to deal with this problem is using the notion of stable intersection.

Let $C_{f}, C_{g}$ be the set of zeroes of two tropical polynomials $f$ and $g$ respectively. Let $P$ be the intersection of the curves, $P=C_{f} \cap C_{g}$. It is possible that $P$ is not the image of an algebraic variety $\widetilde{P}$ by the map $T$. We want to associate, to each $q \in P$ an intersection multiplicity. We will follow the notions of [9] and we will compare them with the subdivisions of 
the associated Newton polygons of the curves in terms of mixed volumes. See [12] to precise the comparison between mixed volumes and intersection of algebraic curves.

Let $C_{f g}=C_{f} \cup C_{g}$. It is easy to check that the union of the two tropical curves is the set of zeroes of the product " $f g$ ". The Newton polygon $\Delta_{f g}$ of $C_{f} \cup C_{g}$ is the Minkowski sum of $\Delta_{f}$ and $\Delta_{g}$. That is:

$$
\Delta_{f g}=\left\{x+y \mid x \in \Delta_{f}, y \in \Delta_{g}\right\}
$$

The subdivision of $\Delta_{f g}$ dual to $C_{f g}$ is a subdivision induced by the subdivisions of $\Delta_{f}, \Delta_{g}$. More concretely, let $q$ be a point in $C_{f g}$, let $\left\{i_{1}, \ldots, i_{n}\right\}$ be the monomials of $f$ where $f(q)$ is attained and let $\left\{j_{1}, \ldots, j_{m}\right\}$ be the monomials of $g$ where $g(q)$ is attained. Then $n \geq 2$ or $m \geq 2$. The monomials where " $f g$ " attains its maximum are $\left\{i_{r} j_{s} \mid 1 \leq r \leq n, 1 \leq s \leq m\right\}$. The Newton polygon of these monomials is the Minkowski sum of the Newton polygons of $\left\{i_{1}, \ldots, i_{n}\right\}$ and $\left\{j_{1}, \ldots, j_{m}\right\}$, each one of these Newton polygons is the cell dual to the cell containing $q$ in $\Delta_{f g}, \Delta_{f}$ and $\Delta_{g}$ respectively. This process covers every cell of dimension 1 and 2 of $\Delta_{f g}$. The zero dimensional cells correspond to points $q$ belonging neither to $C_{f}$ nor to $C_{g}$. Let $i, j$ be the monomials of $f$ and $g$ where the value at $q$ is attained. Then the monomial of " $f g$ " where ( $f g$ " $)(q)$ is attained is $i j$. To sum up, every cell of $\Delta_{f g}$ is naturally the Minkowski sum of a cell $u$ of $f$ and a cell $v$ of $g$. The possible combination of $\operatorname{dimensions}(\operatorname{dim}(u), \operatorname{dim}(v), \operatorname{dim}(u+v))$ are:

- $(0,0,0)$, these cells do not correspond to points of $C_{f g}$.

- $(1,0,1)$, these are edges of $C_{f g}$ that correspond to a maximal segment contained in an edge of $C_{f}$ that does not intersect $C_{g}$.

- $(2,0,2)$, correspond to the vertices of $C_{f g}$ that are vertices of $C_{f}$ that do not belong to $C_{g}$.

- $(1,1,2)$, this combination defines a vertex of $C_{f g}$ which is the unique intersection point of an edge of $C_{f}$ with an edge of $C_{g}$.

- $(1,1,1)$ are the edges of $C_{f g}$ that are the infinite intersection of an edge of $C_{f}$ and an edge of $C_{g}$.

- $(1,2,2)$ corresponds with the vertices of $C_{f g}$ that are a vertex of $C_{g}$ belonging to an edge of $C_{f}$.

- $(2,2,2)$ This is a vertex of $C_{f g}$ which is a common vertex of $C_{f}$ and $C_{g}$.

and the obvious symmetric cases $(0,1,1),(0,2,2)$ and $(2,1,2)$.

If the relative position of $C_{f}, C_{g}$ is generic, then $C_{f g}$ cannot contain any cell of type $(1,1,1),(1,2,2)$ and $(2,2,2)$. That is, the intersection points $q$ of $C_{f}$ and $C_{g}$ are always the unique intersection point of an edge of $C_{f}$ and 
an edge of $C_{g}$. This is the transversal case. The definition of intersection multiplicity, as presented in [9] for these cells $(1,1,2)$ is the following:

Definition 3. Let $q$ be an intersection point of two tropical curves $C_{f}$ and $C_{g}$. Suppose that $q$ is the unique intersection point of an edge $r$ of $C_{f}$ and an edge $s$ of $C_{g}$. Let $\vec{r}$ be the primitive vector in $\mathbb{Z}^{2}$ of the support line of $r$. Let $\vec{s}$ be the corresponding primitive vector of $s$. Let $u$ be the dual edge of $r$ in $\Delta_{f}$ and let $v$ be the dual edge of $s$ in $\Delta_{g}$, we call $m_{u}=\#\left(\bar{u} \cap \mathbb{Z}^{2}\right)-1$ and $m_{v}=\#\left(\bar{v} \cap \mathbb{Z}^{2}\right)-1$ the weight of the edges $r$ and $s$ respectively. The intersection multiplicity is

$$
\operatorname{mult}(q)=\left|m_{u} m_{v}\right| \begin{array}{ll}
\overrightarrow{r_{x}} & \overrightarrow{r_{y}} \\
\overrightarrow{s_{x}} & \overrightarrow{s_{y}}
\end{array} \mid
$$

the absolute value of the determinant of the primitive vectors times the weight of the edges.

If the curves are not in a generic relative position, consider the curve $C_{f}^{v}$ obtained by translation of $C_{f}$ by a vector $v$. If the length of $v$ is sufficiently small, $|v|<\epsilon$ (that is, it is an infinitesimal translation), then every cell of $\Delta_{f^{\prime} g}$ of type $(0,0,0),(1,0,1),(2,0,2)$ and $(1,1,2)$ stays invariant. Furthermore, if the translation is generic (for all but finitely many directions of $v)$, the cells of type $(1,1,1)$ are subdivided into cells of type $(0,0,0)$ and $(0,1,1)$. That is, if two edges intersect in infinitely many points, after the translation, every intersection point will disappear. If $q$ is an intersection point of $C_{f}$ and $C_{g}$ corresponding to a cell of type $(2,1,2)$ or $(2,2,2)$ and the direction of $v$ is generic, this cell is subdivided, after the perturbation, into cells of type $(0,0,0),(1,0,1),(1,1,2),(2,0,2)$. That is, no intersection point is a vertex of $f$ or $g$. However, some transversal intersection points appear instead (of type $(1,1,2)$ ) in a neighborhood of $q$. The intersection multiplicity of $q$ is, in this case, the sum of the intersection multiplicities of the transversal intersection points.

Now we recall the notion of stable intersection of curves (See [9]).

Definition 4. Let $C_{f}, C_{g}$ be two tropical curves. Let $C_{f}^{v}, C_{g}^{w}$ be two small generic translations of $C_{f}, C_{g}$ such that their intersection is finite. The stable intersection $C_{f} \cap_{s t} C_{g}$ of $C_{f}$ and $C_{g}$ is the limit set of intersection points of the translated curves $\lim _{v, w \rightarrow 0}\left(C_{f}^{v} \cap C_{g}^{w}\right)$.

From the previous comments it is clear that

Proposition 5. Let $C_{f}, C_{g}$ be two tropical curves, then the stable intersection of $C_{f}$ and $C_{g}$ is the set of intersection points with positive multiplicity.

This stable intersection has very nice properties. From the definition, it follows that it is continuous under small perturbations on the curves. Moreover, it verifies a Berstein-Koushnirenko Theorem for tropical curves. 
Theorem 6. Let $C_{f}, C_{g}$ be two tropical curves of Newton polygons $\Delta_{f}, \Delta_{g}$. Then the number of stable intersection points, counted with multiplicity is the mixed volumes of the Newton polygons of the curves

$$
\sum_{q \in C_{f} \cap_{s t} C_{g}} m(q)=\mathcal{M}\left(\Delta_{f}, \Delta_{g}\right)=\operatorname{vol}\left(\Delta_{f}+\Delta_{g}\right)-\operatorname{vol}\left(\Delta_{f}\right)-\operatorname{vol}\left(\Delta_{g}\right)
$$

Proof. See [9].

In particular, we have the following alternative definition of intersection multiplicity for plane curves:

Corollary 7. Let $f, g$ be two tropical polynomials of Newton polygons $\Delta_{f}$, $\Delta_{g}$ respectively. Let $q \in \mathcal{T}(f) \cap \mathcal{T}(g)$ be an intersection point. Let $\Lambda_{f}$, $\Lambda_{g}$ be the cells of Subdiv $\left(\Delta_{f}\right)$, Subdiv $\left(\Delta_{g}\right)$ dual to the cells in the curve containing $q$ respectively, then, the tropical intersection multiplicity of $q$ is:

$$
\operatorname{mult}(q)=\mathcal{M}\left(\Lambda_{f}, \Lambda_{g}\right)=\operatorname{vol}\left(\Lambda_{f}+\Lambda_{g}\right)-\operatorname{vol}\left(\Lambda_{f}\right)-\operatorname{vol}\left(\Lambda_{g}\right) .
$$

Proof. From the classification of intersection points, $q$ is an intersection point of multiplicity zero if and only if it belongs to a cell of type $(1,1,1)$ in $C_{f g}$. In this case $\mathcal{M}\left(\Lambda_{f}, \Lambda_{g}\right)=\operatorname{vol}\left(\Lambda_{f}+\Lambda_{g}\right)-\operatorname{vol}\left(\Lambda_{f}\right)-\operatorname{vol}\left(\Lambda_{g}\right)=0$, because an edge has no area. If $q$ is a stable intersection point, let $f=$ " $\sum_{i \in \Delta_{f}} a_{i} x^{i_{1}} y^{i_{2}} ", g=" \sum_{j \in \Delta_{g}} b_{j} x^{j_{1}} y^{j_{2}} "$, let $f_{q}=" \sum_{i \in \Lambda_{f}} a_{i} x^{i} ", g_{q}=$ " $\sum_{j \in \Lambda_{g}} b_{j} x^{j}$ " be truncated polynomials. It follows from the definition that the intersection multiplicity of $q$ only depends in the behaviour of the mixed cell $\Lambda_{f}+\Lambda_{g}$ in the dual subdivision of $\Delta_{f g}$. That is, the intersection multiplicity of $q$ as intersection of $C_{f}$ and $C_{g}$ equals the intersection multiplicity of $q$ as an intersection point of $\mathcal{T}\left(f_{q}\right)$ and $\mathcal{T}\left(g_{q}\right)$. But, by construction, the unique stable intersection point of $\mathcal{T}\left(f_{q}\right)$ and $\mathcal{T}\left(g_{q}\right)$ is $q$ itself. Hence, by Theorem 6, the intersection multiplicity of $q$ is

$$
\mathcal{M}\left(\Lambda_{f}, \Lambda_{g}\right)=\operatorname{vol}\left(\Lambda_{f}+\Lambda_{g}\right)-\operatorname{vol}\left(\Lambda_{f}\right)-\operatorname{vol}\left(\Lambda_{g}\right)
$$

\section{Univariate resultants}

Let us start with the notion of tropical resultant of two univariate polynomials. In algebraic geometry, the resultant of two univariate polynomials is a polynomial that solves the decision problem of determining if both polynomials have a common root.

Definition 8. Let $\tilde{f}=\sum_{i=0}^{n} a_{i} x^{i}, \widetilde{g}=\sum_{j=0}^{m} b_{j} x^{j} \in \mathbb{K}[x]$, where $\mathbb{K}$ is an algebraically closed field. For simplicity, we assume that $a_{0} a_{n} b_{0} b_{m} \neq 0$. 
Let $p$ be the characteristic of $\mathbb{K}$. Then, there is a unique polynomial in $\mathbb{Z} /(p \mathbb{Z})\left[a_{i}, b_{j}\right]$, up to a constant factor, called the resultant, such that it vanishes if and only if $\widetilde{f}$ and $\widetilde{g}$ have a common root.

In the definition, it is asked the polynomials to be of effective degree $n$ and $m$, this is in order to avoid the specialization problems that usually appear when using resultants. But the polynomials are also asked to have order zero. This restriction is demanded for convenience with tropicalization. Recall that the intersection of the varieties with the coordinate hyperplanes is always neglected. Hence, the definition of resultant will take this into account. Moreover, as the polynomials are described by its support, the resultant will not be defined by the degree of the polynomials, but by their support. This approach will be convenient in the next section, when there will be provided a notion of resultant for bivariate polynomials.

Definition 9. Let $I, J$ be two finite subsets of $\mathbb{N}$ of cardinality at least 2 such that $0 \in I \cap J$. That is, the support of two polynomials that do not have zero as a root. Let $R(I, J, \mathbb{K})$ be the resultant of two polynomials with indeterminate coefficients, $f=\sum_{i \in I} a_{i} x^{i}, g=\sum_{j \in J} b_{j} x^{j}$ over the field $\mathbb{K}$.

$$
R(I, J, \mathbb{K}) \in \mathbb{Z} /(p \mathbb{Z})[a, b],
$$

(where $p$ is the characteristic of the field $\mathbb{K}$ ). Let $R_{t}(I, J, \mathbb{K})$ be the tropicalization of $R(I, J, \mathbb{K})$. This is a polynomial in $\mathbb{T}[a, b]$, which is called the tropical resultant of supports $I$ and $J$ over $\mathbb{K}$.

So, our approach is to define the tropical resultant polynomial as the projection of the algebraic polynomial. In this point, one may obtain, for the same support sets $I$ and $J$, different tropical resultants, one for each possible characteristic of $\mathbb{K}$. This is not good, in the sense that tropical geometry should not be determined by the characteristic of the field we have used to define the projection. Hence, one has to take care of what is the common information of these polynomials. The answer is complete: the tropical variety they define is always the same. This variety is the image of any resultant variety over a field $\mathbb{K}$, so it will code the pairs of polynomials with fixed support that have a common root.

Lemma 10. The tropical variety $\mathcal{T}\left(R_{t}(I, J, \mathbb{K})\right)$ does not depend on the field $\mathbb{K}$, but only on the sets $I$ and $J$.

Proof. Let $\mathcal{N}$ be the Newton polytope of the resultant defined over a field $\mathbb{L}$ of characteristic zero, $\mathcal{N} \subseteq \mathbb{R}^{n+m+2}$. It is known that the monomials of $R(I, J, \mathbb{L})$ corresponding to vertices of $\mathcal{N}$ (extreme monomials) have 
always as coefficient \pm 1 (See, for example, [4] or [11]). Hence, the extreme monomials in $R(I, J, \mathbb{K})$ are independent of the characteristic of the field $\mathbb{K}$ and so is $\mathcal{N}$. If $x=\left(x_{1}^{j_{1}}, \ldots, x_{N}^{j_{N}}\right)$ is a monomial of $R(I, J, \mathbb{K})$ that does not correspond to a vertex of $\mathcal{N}$, then $x=\sum \lambda_{i} v_{i}, 0 \leq \lambda_{i} \leq 1$, where $v_{i}=\left(x_{1}^{j_{i, 1}}, \ldots, x_{N}^{j_{i, N}}\right)$ are vertices of $\mathcal{N} \cdot T\left(\operatorname{coeff}\left(v_{i}\right)\right)=T( \pm 1)=0$ and, as coeff $(x)$ is an integer (or an integer $\bmod p$ ), it is contained in the valuation ring, that is, $0 \geq T(\operatorname{coeff}(x)) \in \mathbb{T} \cup\{-\infty\}$. $T(\operatorname{coeff}(x))$ is finite and not zero if and only if we are dealing with a $p$-adic valuation and $p$ divides coeff $(x)$. It is $-\infty$ if and only if the characteristic of $\mathbb{K}$ divides the coefficient. Hence, for any evaluation $w$ of the indeterminates, we have that $T(\operatorname{coeff}(x))+w_{1} j_{1}+\ldots+w_{N} j_{N} \leq w_{1} j_{1}+\ldots+w_{N} j_{N}=\sum \lambda_{i} v_{i}(w) \leq$ $\max _{i}\left\{v_{i}(w)\right\}$, where $v_{i}(w)=w_{1} j_{i, 1}+\ldots+w_{N} j_{i, N}$. It follows that the maximum of the piecewise affine function $R_{t}(I, J, \mathbb{K})$ is never attained in the monomial $x$ alone and that $x$ does not induce any subdivision in the cell it is contained. Thus, this monomial does not add anything to the tropical variety defined by $R_{t}(I, J, \mathbb{K})$. The tropical hypersurface $\mathcal{T}\left(R_{t}(I, J, \mathbb{K})\right)$ is, as a polyhedral complex, dual to the subdivision of $\mathcal{N}$ induced by $R_{t}(I, J, \mathbb{K})$ (cf. [8]). In this case, the subdivision of $\mathcal{N}$ induced by the tropical polynomial is $\mathcal{N}$ itself. So $\mathcal{T}\left(R_{t}(I, J, \mathbb{K})\right)$ is always the polyhedral complex dual to $\mathcal{N}$ centered at the origin. This complex is independent of $\mathbb{K}$.

Hence, fixed two supports $I, J$, there may be different tropical polynomials that can be called the resultant of polynomials of support $I$ and $J$. However, the variety all of them define is always the same, so there is a good notion of resultant variety. Now we prove that the resultant variety $\mathcal{T}\left(R_{t}(I, J, \mathbb{K})\right)$ has the same geometric meaning than the algebraic resultant variety.

Lemma 11. Let $I, J$ be two support subsets as before. Let $f=" \sum_{i \in I} a_{i} x^{i "}$, $g=" \sum_{j \in J}^{m} b_{j} x^{j}$ " be two univariate tropical polynomials of support I and $J$. Then, $f$ and $g$ have a common tropical root if and only if the point $\left(a_{i}, b_{j}\right)$ belongs to the variety defined by $R_{t}(I, J, \mathbb{K})$.

Proof. Suppose that $\left(a_{i}, b_{j}\right)$ belongs to $R_{t}(I, J, \mathbb{K})$. By Theorem 1 , we can compute an element $\left(\widetilde{a}_{i}, \widetilde{b}_{j}\right)$ in the variety defined by $R(I, J, \mathbb{K})$. In this case, $\widetilde{f}=\sum_{i \in I} \widetilde{a}_{i} x^{i}$ and $\widetilde{g}=\sum_{j \in J} \widetilde{b}_{j} x^{j}$ are lifts of $f$ and $g$. That is, $T(\widetilde{f})=f$, $T(\widetilde{g})=g$. Moreover, their coefficients belong to the algebraic resultant, so the algebraic polynomials have a common root $\widetilde{q}$ that is non zero by construction $(0 \in I \cap J)$. Projecting to the tropical space, $f$ and $g$ have a common root $T(\widetilde{q})$. Conversely, if $f$ and $g$ have a common root $q$, we may take any lift $\widetilde{g}=\sum_{j \in J} \widetilde{b}_{j} x^{j}$ of $g$. Then, by Theorem 1, we may lift $q$ to a root $\widetilde{q}$ of $\widetilde{g}$. Finally, note that the coefficients of $f$ belong to the hyperplane defined by the equation $\sum_{i \in I} z_{i} q^{i}$, so it can be lifted to an algebraic 
solution $\widetilde{a}$ of the affine equation $\sum_{i \in I} z_{i} \widetilde{q}^{i}$, the polynomial $\widetilde{f}=\sum_{i \in I} \widetilde{a}_{i} x^{i}$ projects onto $f$ and has $\widetilde{q}$ as a root. By construction, $\widetilde{f}, \widetilde{g}$ share a common root $\widetilde{q}$, hence, their coefficients $\left(\widetilde{a}_{i}, \widetilde{b}_{j}\right)$ belong to the algebraic resultant variety. Projecting again, the coefficient vector $\left(a_{i}, b_{j}\right)$ of $f$ and $g$ belong to the tropical resultant.

This Lemma about the geometric meaning of the resultant also shows that the variety defined by $R_{t}(I, J, \mathbb{K})$ does not depend on the field $\mathbb{K}$. At least as a set of points, because the tropical characterization of two tropical polynomials having a common root does not depend on the field $\mathbb{K}$.

Example 12. Consider the easiest nonlinear case, $I=J=\{0,1,2\}$, the resultant of two quadratic polynomials. If $f=a+b x+c x^{2}, g=p+q x+r x^{2}$, the algebraic resultant in characteristic zero is $R_{0}=r^{2} a^{2}-2 r a c p+c^{2} p^{2}-$ $q r b a-q b c p+c q^{2} a+p r b^{2}$ and, over a characteristic 2 field it is $R_{2}=r^{2} a^{2}+$ $c^{2} p^{2}+q r b a+q b c p+c q^{2} a+p r b^{2}$. If $\operatorname{char}(k) \neq 2$, the tropical polynomial is $P_{1}=" 0 r^{2} a^{2}+0 r a c p+0 c^{2} p^{2}+0 q r b a+0 q b c p+0 c q^{2} a+0 p r b^{2} "$. If $\operatorname{char}(\mathbb{K})=0$ and $\operatorname{char}(k)=2$, the tropical polynomial is $P_{2}=$ " $0 r^{2} a^{2}+(-1) r a c p+$ $0 c^{2} p^{2}+0 q r b a+0 q b c p+0 c q^{2} a+0 p r b^{2} "$. Finally, if $\operatorname{char}(k)=\operatorname{char}(\mathbb{K})=2$ then the tropical polynomial is $P_{3}=" 0 r^{2} a^{2}+0 c^{2} p^{2}+0 q r b a+0 q b c p+0 c q^{2} a+$ $0 p r b^{2}$ ". The unique difference among these polynomials is the term racp. This monomial lies in the convex hull of the monomials $r^{2} a^{2}$ and $c^{2} p^{2}$ and it does not define a subdivision because its tropical coefficient is always $\leq 0$. The piecewise affine functions $\max \{2 r+2 a, r+a+c+p, 2 c+2 p\}$, $\max \{2 r+2 a,-1+r+a+c+p, 2 c+2 p\}$ and $\max \{2 r+2 a, 2 c+a p\}$ are the same. So the three polynomials define the same tropical variety.

\section{Resultant of two curves}

In this section, the notion of univariate resultant is extended to the case where the polynomials are bivariate.

Definition 13. Let $\widetilde{f}$ and $\widetilde{g}$ be two bivariate polynomials. In order to compute the algebraic resultant with respect to $x$, we can rewrite them as polynomials in $x$.

$$
\widetilde{f}=\sum_{i \in I} \widetilde{f}_{i}(y) x^{i}, \quad \widetilde{g}=\sum_{j \in J} \widetilde{g}_{j}(y) x^{j}
$$

where

$$
\widetilde{f}_{i}=\sum_{k=o_{i}}^{n_{i}} A_{i k} t^{-\nu_{i k}} y^{k}, \quad \widetilde{g}_{j}=\sum_{q=r_{j}}^{m_{j}} B_{j q} t^{-\eta_{j q}} y^{q}
$$

and $A_{i k}, B_{j q}$ are elements of valuation zero. 
Let $P\left(a_{i}, b_{j}, \mathbb{K}\right)=R(I, J, \mathbb{K}) \in \mathbb{Z} /(p \mathbb{Z})\left[a_{i}, b_{j}\right]$ be the algebraic univariate resultant of supports $I, J$. The algebraic resultant of $\widetilde{f}$ and $\widetilde{g}$ is the polynomial $P\left(\widetilde{f}_{i}, \widetilde{g}_{j}, \mathbb{K}\right) \in \mathbb{K}[y]$. Analogously, let $f=T(\widetilde{f}), g=T(\widetilde{g})$, $f=" \sum_{i \in I} f_{i}(y) x^{i} ", g=" \sum_{j \in J} g_{j}(y) x^{j} "$, where

$$
f_{i}=" \sum_{k=o_{i}}^{n_{i}} \nu_{i k} y^{k} ", \quad g_{j}=" \sum_{q=r_{j}}^{m_{j}} \eta_{j q} y^{q} " .
$$

Let $P_{t}\left(a_{i}, b_{j}, \mathbb{K}\right)=R_{t}(I, J, \mathbb{K}) \in \mathbb{T}\left[a_{i}, b_{j}\right]$ be the tropical resultant of supports $I$ and $J$. Then, the polynomial $P_{t}\left(f_{i}, g_{j}, \mathbb{K}\right) \in \mathbb{T}[y]$ is the tropical resultant of $f$ and $g$.

Again, we have different tropical resultant polynomials, one for each possible characteristic of the fields $\mathbb{K}$ and $k$. We want to check that this notion of tropical resultant also has a geometric meaning. In the algebraic setting, the roots of the resultant $P\left(\widetilde{f}_{i}, \widetilde{g}_{j}, \mathbb{K}\right)$ are the possible $y$-th values of the intersection points of the curves defined by $\widetilde{f}$ and $\widetilde{g}$. This is not the case of the tropical resultant, because $P_{t}\left(f_{i}, g_{j}, \mathbb{K}\right)$ only has finitely many tropical roots, while the intersection $\mathcal{T}(f) \cap \mathcal{T}(g)$ may have infinitely many points and there may be infinitely many possible values of the $y$-th coordinates. Again, this indetermination is avoided with the notion of stable intersection. We will prove that the roots of $P_{t}\left(f_{i}, g_{j}, \mathbb{K}\right)$ are the possible $y$-th values of the stable intersection $\mathcal{T}(f) \cap_{s t} \mathcal{T}(g)$. This will be made in several steps, the first one is to check that $T\left(V\left(P\left(\widetilde{f}_{i}, \widetilde{g}_{j}, \mathbb{K}\right)\right)\right)=\mathcal{T}\left(P_{t}\left(f_{i}, g_{j}, \mathbb{K}\right)\right)$, provided that $A_{i k}, B_{j q}$ are residually generic. Sometimes, for technical reasons, it is better to work with an affine representation of the polynomials. The set of polynomials $\widetilde{f}=$ $\sum_{i \in I} a_{i} x^{i^{1}} y^{i^{2}}$ of fixed support $I$ is an open subspace (a torus) of a projective space. The projective coordinates of $\tilde{f}$ are its coefficients $\left[a_{i}: i \in I\right]$. We may fix an index $i_{0} \in I$. Then, the affine representation of $\widetilde{f}$ with respect to this index is obtained by setting $a_{i_{0}}=1$ and $a_{i_{j}}=a_{i_{j}} / a_{i_{0}}$. We prove that this dehomogenization process is also compatible with tropicalization. That is, if we divide each algebraic coefficient $A_{i k} t^{-\nu_{i k}}$ and $B_{j q} t^{-\eta_{j q}}$ by $A_{i_{0} k_{0}} t^{-\nu_{i_{0}} k_{0}}$ and $B_{j_{0} q_{0}} t^{-\eta_{j_{0} q_{0}}}$ respectively and substitute each coefficient $\nu_{i k}, \eta_{j q}$ of the tropical polynomials $f$ and $g$ by $\nu_{i k}-\nu_{i_{0} k_{0}}=$ " $\nu_{i k} / \nu_{i_{0} k_{0}}$ " and $\eta_{i q}-\eta_{i_{0} q_{0}}$ respectively, still we have that $T\left(V\left(P\left(\widetilde{f}_{i}, \widetilde{g}_{j}, \mathbb{K}\right)\right)\right)=\mathcal{T}\left(P_{t}\left(f_{i}, g_{j}, \mathbb{K}\right)\right)$.

Lemma 14. Let $\widetilde{f}=\sum_{i \in I} \widetilde{f}_{i} x^{i}, \widetilde{g}=\sum_{j \in J} \widetilde{g}_{j} x^{j} \in \mathbb{K}[x, y]$, where the coefficients are

$$
\widetilde{f}_{i}=\sum_{k=o_{i}}^{n_{i}} A_{i k} t^{-\nu_{i k}} y^{k}, \quad \widetilde{g}_{j}=\sum_{q=r_{j}}^{m_{j}} B_{j q} t^{-\eta_{j q}} y^{q}
$$


and let $f=\sum_{i \in I} f_{i}(y) x^{i}, g=\sum_{j \in J} g_{j}(y) x^{j}$,

$$
f_{i}=" \sum_{k=o_{i}}^{n_{i}} \nu_{i k} y^{k} ", \quad g_{j}=" \sum_{q=r_{j}}^{m_{j}} \eta_{j q} y^{q} "
$$

be the corresponding tropical polynomials. Suppose that $A_{i k}, B_{j q}$ are residually generic. Then $T\left(V\left(P\left(\widetilde{f}_{i}, \widetilde{g}_{j}, \mathbb{K}\right)\right)\right)=\mathcal{T}\left(P_{t}\left(f_{i}, g_{j}, \mathbb{K}\right)\right)$.

Proof. First, we suppose that $\operatorname{char}(k)=0$. In general, the composition of polynomials does not commute with tropicalization, because, in the algebraic case, there can be a cancellation of terms when performing the substitution that does not occur in the tropical case. Recall that, by the nature of tropical operations, a cancellation of terms in the tropical development of the polynomial never happens. So, we have to check that there is never a cancellation of terms in the algebraic setting. First, it is proved that there is no cancellation of monomials when substituting the variables by polynomials without dehomogenizing. $P\left(a_{i}, b_{j}, \mathbb{K}\right)$ is homogeneous in the set of variables $a_{i}$ and in the set of variables $b_{j}$. As the substitution is linear in the variables $A_{i k}$ and $B_{j q}, P\left(\widetilde{f}_{i}, \widetilde{g}_{j}, \mathbb{K}\right)$ is homogeneous in $A_{i j}$ and $B_{j q}$. If we have two different terms $T_{1}, T_{2}$ of $P\left(a_{i}, b_{j}, \mathbb{K}\right)$, then there is a variable with different exponent in both terms. Assume for simplicity that this variable is $a_{1}$ with degrees $d_{1}$ and $d_{2}$ respectively. After the substitution, the monomials obtained by expansion of $T_{1}$ are homogeneous of degree $d_{1}$ in the set of variables $A_{1 k}$ and the monomials coming from $T_{2}$ are homogeneous of degree $d_{2}$ in the variables $A_{1 k}$. Thus, it is not possible to have a cancellation of terms and we can conclude that the homogeneous polynomial projects onto the tropical homogeneous polynomial.

In the case we dehomogenize $\widetilde{f}$ and $\widetilde{g}$ with respect to the indices $\left(i_{0} k_{0}\right)$, $\left(j_{0} q_{0}\right)$ respectively. By the homogeneous case, we can suppose that all the variables $a_{i} \neq a_{i_{0}}$ and $b_{j} \neq b_{j_{0}}$ in $P\left(a_{i}, b_{j}, \mathbb{K}\right)$ have already been substituted by the polynomials $\tilde{f}_{i}$ and $\widetilde{g}_{j}$ respectively. The only possibility to have a cancellation of terms is if there are two monomials of the form $X a_{i_{0}}^{d_{1}} b_{j_{0}}^{d_{2}}, X a_{i_{0}}^{d_{3}} b_{j_{0}}^{d_{4}}$ with $d_{1}+d_{2}=d_{3}+d_{4}$ and $X$ is a monomial in the variables $A_{i k}, B_{j q}$. But, as the polynomial is multihomogeneous in $A$ and $B$, it must happen that $d_{1}=d_{3}$ and $d_{2}=d_{4}$. That is, the original monomials were the same. So, a cancellation of terms is not possible and the dehomogenized polynomial projects into the dehomogenized tropical polynomial. In particular, $T\left(V\left(P\left(\widetilde{f}_{i}, \widetilde{g}_{j}, \mathbb{K}\right)\right)\right)=\mathcal{T}\left(P_{t}\left(f_{i}, g_{j}, \mathbb{K}\right)\right)$.

Now suppose that $\operatorname{char}(k)=p>0$. In this case, it is not necessarily true that the tropicalization of the algebraic resultant is the tropical resultant. But we are going to check that the monomials where these 
two tropical polynomials differ do not add anything to the tropical variety $\mathcal{T}\left(f_{i}, g_{i}, \mathbb{K}\right)$. So, we are going to compare the monomials in $P\left(\widetilde{f}_{i}, \widetilde{g}_{j}, \mathbb{K}\right)$ and $P_{t}\left(f_{i}, g_{j}, \mathbb{K}\right)$. The support of both polynomials is contained in the support of $P_{t}\left(f_{i}, g_{j}, \mathbb{L}\right)$, where $\mathbb{L}$ is an equicharacteristic zero field. The first potential difference in the monomials are those obtained by expansion of a monomial $m$ of the univariate resultant $P\left(a_{i}, b_{j}, \mathbb{K}\right)=R(I, J, \mathbb{K})$ whose coefficient has valuation in $[-\infty, 0)$. That is, $p$ divides coeff $(m)$. It happens that $m$ is never a extreme monomial. That is, $m=\sum_{l} \lambda_{l} v_{l}, 0 \leq \lambda_{l} \leq 1$ and $v_{i}$ are extreme monomials. So, for every $r$, coeff $(m)+m\left(f_{i}(r), g_{j}(r)\right) \leq$ $m\left(f_{i}(r), g_{j}(r)\right)=\sum_{l} \lambda_{l} v_{l}\left(f_{i}(r), g_{j}(r)\right) \leq \max \left\{v_{l}\left(f_{i}(r), g_{j}(r)\right)\right\}$. Hence, the monomials of $m\left(f_{i}(y), g_{j}(y)\right)$ never add anything to the tropical variety defined by $P\left(\widetilde{f}_{i}, \widetilde{g}_{j}, \mathbb{K}\right)$, because they are never greater than the monomials that appear by the extreme monomials. The other source of potential differences in the monomials is the decreasing of the tropicalization of some terms of the power $\left(\sum_{k=o_{i}}^{n_{i}} A_{i k} t^{-\nu_{i k}} y^{k}\right)^{N}$ due to some combinatorial coefficient $\left(\begin{array}{l}N \\ m\end{array}\right)$ divisible by $p$. But, in the tropical context, it happens that

$$
\left(" \sum_{k=o_{i}}^{n_{i}} \nu_{i k} y^{k} "\right)^{N}=" \sum_{k=o_{i}}^{n_{i}} \nu_{i k}^{N} y^{k N "}
$$

as piecewise affine functions. The rest of the terms in the expansion do not contribute anything to the tropical variety. The only terms that may play a role are $\nu_{i k}^{N}, \eta_{j q}^{M}$. So, even if the tropicalization of the polynomials $P(I, J, \mathbb{K})$ depends on the algebraic field $\mathbb{K}$, the tropical variety they define is always the same and it is the tropical variety defined by $P_{t}\left(f_{i}, g_{j}, \mathbb{K}\right)$, including the weight of the cells.

So, the previous Lemma provides a notion of tropical resultant for bivariate polynomials with respect to one variable. They also prove that this polynomials define the same variety as the projection of the algebraic resultant in the generic case. Our next goal is to provide a geometric meaning to the roots of the tropical resultant in terms of the stable intersection of the curves.

\section{Computation of the stable intersection}

Let $f$ be a tropical polynomial of support $I$ defining a curve, let $\Delta_{f}$ be the convex hull of $I$. By Proposition 2, the coefficients of $f$ induce a regular subdivision in $\Delta_{f}$ dual to $f$. This subdivision is essential in the definition of tropical multiplicity and stable intersection for the case of curves. Next, it is proved that, for sufficiently generic lifts $\widetilde{f}$ and $\widetilde{g}$, their intersection points correspond with stable intersection points of $f$ and $g$. 
Lemma 15. Let $f$ and $g$ be two tropical polynomials in two variables. Let $L$ be its stable intersection. Then, for any two lifts $\widetilde{f}, \widetilde{g}$ such that their coeffcients are residually generic, the intersection of the algebraic curves projects into the stable intersection.

$$
T(\tilde{f} \cap \widetilde{g}) \subseteq \mathcal{T}(f) \cap_{s t} \mathcal{T}(g)
$$

Proof. If every intersection point of $f$ and $g$ is stable, then there is nothing to prove. Let $q$ be a non stable intersection point. This means that $q$ belongs to the relative interior of two parallel edges of $\mathcal{T}(f)$ and $\mathcal{T}(g)$. The residual polynomials $\widetilde{f}_{q}$ and $\widetilde{g}_{q}$ can be written (after multiplication by a suitable monomial) as $\widetilde{f}_{q}=\sum_{i=0}^{n} \alpha_{i}\left(x^{r} y^{s}\right)^{i}, \widetilde{g}_{q}=\sum_{j=0}^{m} \beta_{i}\left(x^{r} y^{s}\right)^{j}$. If $\tilde{f}, \widetilde{g}$ have a common point projecting into $q$ then there is an algebraic relation among their residual coefficients. Namely, the resultant of the polynomials $\sum_{i=0}^{n} \alpha_{i} z^{i}$, $\sum_{j=0}^{m} \beta_{i} z^{j}$ with respect to $z$ must vanish. If the residual coefficients of $\widetilde{f}, \widetilde{g}$ do not belong to the resultant defined by each non stable intersection cell, the intersection in the torus of $\widetilde{f}, \widetilde{g}$ projects into the stable intersection of $f$ and $g$.

So, there is a natural relation between the stable intersection of two tropical curves $f$ and $g$ and the intersection of two generic lifts $\widetilde{f}$ and $\widetilde{g}$ of the curves. On the other hand, the intersection of two generic lifts can be determined by the algebraic resultant of the defining polynomials. Applying tropicalization, this relationship links the notion of stable intersection with the resultants. To achieve a true bijection between the roots of the resultant and the intersection points of the curves, it is used the relationship between the tropical and algebraic resultants. So, one needs to concrete the generality conditions for the values values $A_{i k}, B_{j q}$ that makes Lemma 14 hold. Next Proposition shows how to compute the residually conditions for the compatibility of the resultant.

Proposition 16. Let $\widetilde{f}, \widetilde{g} \in \mathbb{K}[x, y]$. Then, there is a finite set of nonzero polynomials in the principal coefficients of the coefficients of $\widetilde{f}, \widetilde{g}$, that depends only on the tropicalization $f$ and $g$ such that, if no one of them vanishes, then

$$
T\left(\operatorname{Res}_{x}(\tilde{f}, \widetilde{g})\right)=\mathcal{T}(R(I, J, \mathbb{K})(f, g)) .
$$

Where $R(I, J, \mathbb{K})(f, g)$ is the evaluation of the tropical resultant of supports $I$ and $J$ in the coefficients of $f$ and $g$ as polynomials over $x$.

Proof. Write $\widetilde{f}=" \sum_{i, k} \widetilde{a}_{i k} x^{i} y^{k} ", \widetilde{g}=" \sum_{j, q} \widetilde{b}_{j q} x^{j} y^{q} "$, and take $\operatorname{Pc}\left(\widetilde{a}_{i k}\right)=$ $\alpha_{i k}, \operatorname{Pc}\left(\widetilde{b}_{j q}\right)=\beta_{j q}, T\left(\widetilde{a}_{i k}\right)=a_{i k}, T\left(\widetilde{b}_{j q}\right)=b_{j q}, f=" \sum_{i, k} a_{i k} x^{i} y^{k} ", g=$ " $\sum_{j, q} b_{j q} x^{j} y^{q}$ ". Let $I, J$ be the support of $f$ and $g$ with respect to $x$. 
Consider both resultants

$$
R(I, J, \mathbb{K})(\tilde{f}, \widetilde{g})=\sum_{r=0}^{N} \widetilde{h}_{r} y^{r} \quad \text { and } \quad R_{t}(I, J, \mathbb{K})(f, g)=" \sum_{r=0}^{N} h_{r} y " \text { " }
$$

It happens that $T\left(\widetilde{h}_{r}\right) \leq h_{r}$ and the equality holds if and only if the term $\gamma_{r}(\alpha, \beta) t^{-h_{r}}$ of $\widetilde{h}_{r}$ is different from 0 . As in the generic case the resultant projects correctly by Lemma 14 , the polynomials $\gamma_{r}$ corresponding to vertices of the subdivision of the Newton polytope of the resultant polynomial (that in this case is a segment) are nonzero polynomials in $k\left[\alpha_{i k}, \beta_{j q}\right]$. If no one of them vanish, the resultant tropicalizes correctly.

With all these results we are ready to prove our main result, we can provide a bijection between the stable intersection of two tropical curves and the intersection of two generic lifts of the curves. Moreover, sufficient residual conditions for the genericity can be explicitly computed.

Theorem 17. Let $\tilde{f}, \widetilde{g} \in \mathbb{K}[x, y]$. Then, it can be computed a finite set of polynomials in the principal coefficients of $\widetilde{f}, \widetilde{g}$ depending only on their tropicalization $f, g$ such that, if no one of them vanishes, the tropicalization of the intersection of $\widetilde{f}, \widetilde{g}$ is exactly the stable intersection of $f$ and $g$. Moreover, the multiplicities are conserved.

$$
\sum_{\substack{\widetilde{q} \in \tilde{f} \cap \widetilde{g} \\ T(\widetilde{q})=q}} \operatorname{mult}(\widetilde{q})=\operatorname{mult}_{t}(q)
$$

Proof. Proposition 16 provides a set $S$ of polynomials in the principal coefficients of $\widetilde{f}$ and $\widetilde{g}$ such that, if no one vanishes, the algebraic resultants $\operatorname{Res}_{x}(\widetilde{f}, \widetilde{g})$ and $\operatorname{Res}_{y}(\widetilde{f}, \widetilde{g})$ define the same tropical varieties as $\operatorname{Res}_{x}(f, g)$ and $\operatorname{Res}_{y}(f, g)$. These two resultants define a finite set $P$ that contains the stable intersection. The problem is that, in the tropical case, it is possible that the intersection of $P$ with both curves may be strictly larger than the stable intersection of the curves, see Example 19. So, we need another polynomial in order to discriminate the points in this intersection that are not stable points. Take $a$, any natural number such that the affine function $x-a y$ is injective in the finite set $P$. Make the monomial change of coordinates $z=x y^{-a}$. The polynomial $\operatorname{Res}_{y}\left(\widetilde{f}\left(z y^{a}, y\right), \widetilde{g}\left(z y^{a}, y\right)\right)=\widetilde{R}(z)=\widetilde{R}\left(x y^{-a}\right)$ encodes the values $x y^{-a}$ of the common roots of $\widetilde{f}$ and $\widetilde{g}$. We add to the set $S$ the restrictions in the principal coefficients of this resultant to be compatible with tropicalization according to Proposition 16. These values $x y^{-a}$ of the algebraic intersection points correspond with the possible values $x-a y$ of the tropicalization of the roots. As the linear function is injective 
in $P$, then $\mathcal{T}(f) \cap \mathcal{T}(g) \cap \mathcal{T}\left(\operatorname{Res}_{x}(f, g)\right) \cap \mathcal{T}\left(\operatorname{Res}_{y}(f, g)\right) \cap \mathcal{T}(R(" x y$ "a" $))$ is exactly the tropicalization of the intersection points of any system $(\widetilde{f}, \widetilde{g})$ verifying the restrictions of $S$. By, Lemma 15, this set is contained in the stable intersection of $f$ and $g$.

To prove that the multiplicities are conserved, consider the field $\mathbb{K}=$ $\mathbb{C}\left(\left(t^{\mathbb{R}}\right)\right)$ of generalized Puiseux series, in this case

$$
\sum_{\substack{\widetilde{q} \in \tilde{f} \cap \widetilde{g} \\ T(\widetilde{q})=q}} \operatorname{mult}(\widetilde{q}) \leq \operatorname{mult}_{t}(q) .
$$

because the sum on the left is bounded by the mixed volume of the residual polynomials $\widetilde{f}_{q}, \widetilde{g}_{q}$ over $q$ by Bernstein-Koushnirenko Theorem (c.f. [1, 7, 10]). This mixed volume is, by definition, the tropical multiplicity of $q$ on the right. On the other hand, the sum on the left is, over any field, the sum of the multiplicities of the algebraic roots of $\widetilde{R}\left(x y^{-a}\right)$ projecting onto $q$. By the previous results on the correct projection of the resultant, this multiplicity does not depend on $\mathbb{K}$, because it is the degree minus the order of the residual polynomial $R\left(x y^{-a}\right)_{q_{x}-a q_{y}}$, or, equivalently, the multiplicity of $q$ as a root of $T\left(R\left(x y^{-a}\right)\right)$. Moreover, this multiplicity is the mixed volume of the residual polynomials over $q$. That is, the inequality

$$
\sum_{\substack{\widetilde{q} \in \tilde{f} \cap \widetilde{g} \\ T(\widetilde{q})=q}} \operatorname{mult}(\widetilde{q}) \leq \operatorname{mult}_{t}(q)
$$

holds for any field. The total number of roots of $\widetilde{f}$ and $\widetilde{g}$ counted with multiplicities in the torus equals the sum of multiplicities of the stable roots of $f$ and $g$, because, in both cases, this is the degree minus the order of $R\left(x y^{-a}\right)$. From this, we conclude that

$$
\sum_{\substack{\widetilde{q} \in \widetilde{f} \cap \widetilde{g} \\ T(\widetilde{q})=q}} \operatorname{mult}(\widetilde{q})=\operatorname{mult}_{t}(q)
$$

Hence, the projection of the intersection of $\widetilde{f}$ and $\widetilde{g}$ is exactly the stable intersection.

Along the proof of the Theorem we have proved the following result, that asserts that the tropical resultant of two tropical curves has a geometric meaning analogous to the algebraic resultant.

Corollary 18. Let $f, g \in \mathbb{T}[x, y]$ be two tropical polynomials. Let $h(y) \in$ $\mathbb{T}[y]$ be a tropical resultant of $f$ and $g$ with respect to the $x$ variable. Then, the tropical roots of $h$ are exactly the $y$-th coordinates of the stable intersection of $f$ and $g$. 
Example 19. Consider $f=g=" 0+1 x+1 y+1 x y+0 x^{2}+0 y^{2} "$, two conics. Their stable intersection is the set $\{(-1,-1),(0,1),(1,0),(0,0)\}$. Compute the resultants: $\operatorname{Res}_{x}(f, g)=$ " $0+1 y+1 y^{2}+1 y^{3}+0 y$ ", by symmetry $\operatorname{Res}_{y}(f, g)=" 0+1 x+1 x^{2}+1 x^{3}+0 x^{4} "$. Their roots are the lines $y=-1$, $y=0, y=1$ and $x=-1, x=0, x=1$ respectively. In both cases the multiplicity of the roots -1 and 1 is 1 , while the multiplicity of 0 is 2 . The intersection of this lines and the two curves gives the four stable points plus $(-1,1)$ and $(1,-1)$. We need another resultant that discriminates the points. See Figure 1. Take $x-3 y$, the first affine function $x-a y$ that is injective over these points. $f(" z y) ", y)=" 0+1 y+0 y^{2}+1 y^{3} z+1 y^{4} z+0 y^{6} z^{2} "$. $\operatorname{Res}_{y}\left(f\left(" z y^{3} ", y\right), g\left(" z y^{3 "}, y\right)\right)=" 6 z^{8}+9 z^{9}+9 z^{10}+8 z^{11}+6 z^{12}$ ". Its roots are $0,1,2,-3$, all with multiplicity 1 . It is easy to check now that the intersection of the two curves and the three resultants is exactly the stable intersection. The two extra points take the values $-4,4$ in the monomial " $x y^{-3 ",}$ moreover, every point has intersection multiplicity equal to one.

Two generic lifts of the cubics are of the form:

$$
\begin{aligned}
& \tilde{f}=a_{1}+a_{x} t^{-1} x+a_{y} t^{-1} y+a_{x y} t^{-1} x y+a_{x x} x^{2}+a_{y y} y^{2} \\
& \widetilde{g}=c_{1}+c_{x} t^{-1} x+c_{y} t^{-1} y+c_{x y} t^{-1} x y+c_{x x} x^{2}+c_{y y} y^{2}
\end{aligned}
$$

The residual conditions for the compatibility of the algebraic and tropical resultant with respect to $x$ are:

$$
\begin{aligned}
& -\gamma_{x y} \gamma_{x x} \alpha_{x y} \alpha_{y y}-\gamma_{x y} \alpha_{x y} \alpha_{x x} \gamma_{y y}+\gamma_{x y}^{2} \alpha_{x x} \alpha_{y y}+\gamma_{y y} \gamma_{x x} \alpha_{x y}^{2} \\
& -\gamma_{x} \gamma_{x x} \alpha_{x} \alpha_{1}-\gamma_{x} \alpha_{x} \alpha_{x x} \gamma_{1}+\gamma_{1} \gamma_{x x} \alpha_{x}^{2}+\alpha_{x x} \gamma_{x}^{2} \alpha_{1} \\
& \gamma_{y} \gamma_{x x} \alpha_{x}^{2}-\gamma_{x} \gamma_{x x} \alpha_{x} \alpha_{y}+\alpha_{x x} \gamma_{x}^{2} \alpha_{y}-\gamma_{x} \alpha_{x} \alpha_{x x} \gamma_{y} \\
& -\gamma_{x y} \alpha_{x y} \alpha_{x x} \gamma_{y}+\gamma_{y} \gamma_{x x} \alpha_{x y}^{2}-\gamma_{x y} \gamma_{x x} \alpha_{x y} \alpha_{y}+\gamma_{x y}^{2} \alpha_{x x} \alpha_{y}
\end{aligned}
$$

For the resultant with respect to $y$, the compatibility conditions are:

$$
\begin{aligned}
& -\gamma_{y} \gamma_{y y} \alpha_{y} \alpha_{1}-\gamma_{y} \alpha_{y} \alpha_{y y} \gamma_{1}+\gamma_{1} \gamma_{y y} \alpha_{y}^{2}+\gamma_{y}^{2} \alpha_{y y} \alpha_{1}, \\
& \gamma_{x} \gamma_{y y} \alpha_{y}^{2}-\gamma_{y} \alpha_{y} \alpha_{y y} \gamma_{x}+\gamma_{y}^{2} \alpha_{y y} \alpha_{x}-\gamma_{y} \gamma_{y y} \alpha_{y} \alpha_{x}, \\
& \gamma_{x y}^{2} \alpha_{y y} \alpha_{x}+\gamma_{x} \gamma_{y y} \alpha_{x y}^{2}-\gamma_{x y} \gamma_{y y} \alpha_{x y} \alpha_{x}-\gamma_{x y} \alpha_{x y} \alpha_{y y} \gamma_{x}, \\
& -\gamma_{x y} \gamma_{x x} \alpha_{x y} \alpha_{y y}-\gamma_{x y} \alpha_{x y} \alpha_{x x} \gamma_{y y}+\gamma_{x y}^{2} \alpha_{x x} \alpha_{y y}+\gamma_{y y} \gamma_{x x} \alpha_{x y}^{2} .
\end{aligned}
$$

Finally, the third resultant is a degree twelve polynomial in the variable $z$. The residual conditions for its compatibility with the tropical resultant are:

$$
\begin{aligned}
& 2 \gamma_{y y}^{2} \gamma_{x x} \alpha_{x y}^{3} \alpha_{y y} \gamma_{y} \alpha_{y} \gamma_{1} \alpha_{x x} \gamma_{x y}-\gamma_{y y}^{2} \gamma_{x x}^{2} \alpha_{x y}^{4} \alpha_{y y} \gamma_{y} \alpha_{y} \gamma_{1}-2 \gamma_{y y}^{2} \alpha_{x y} \gamma_{x y}^{3} \alpha_{x x}^{2} \\
& \alpha_{y}^{2} \gamma_{1} \alpha_{y y}+\gamma_{x y}^{4} \alpha_{x x}^{2} \gamma_{y y} \alpha_{y y}^{2} \alpha_{y}^{2} \gamma_{1}-\gamma_{x y}^{4} \alpha_{x x}^{2} \gamma_{y y} \alpha_{y y}^{2} \alpha_{y} \gamma_{y} \alpha_{1}+\gamma_{y y}^{2} \alpha_{x y}^{2} \alpha_{y y} \gamma_{y}^{2} \\
& \alpha_{x x}^{2} \gamma_{x y}^{2} \alpha_{1}-\gamma_{x y}^{2} \gamma_{x x}^{2} \alpha_{x y}^{2} \alpha_{y y}^{3} \gamma_{y} \alpha_{y} \gamma_{1}-2 \gamma_{y y} \alpha_{x y} \gamma_{x y}^{3} \alpha_{x x}^{2} \alpha_{y y}^{2} \gamma_{y}^{2} \alpha_{1}+2 \gamma_{y y}^{2} \gamma_{x x}^{2} \\
& \alpha_{x y}^{3} \gamma_{x y} \alpha_{y} \gamma_{y} \alpha_{y y} \alpha_{1}-2 \gamma_{y y}^{2} \gamma_{x x}^{2} \alpha_{x y}^{3} \gamma_{x y}^{2} \alpha_{y}^{2} \gamma_{1} \alpha_{y y}-\gamma_{x y}^{2} \gamma_{x x}^{2} \alpha_{x y}^{2} \gamma_{y y} \alpha_{y y}^{2} \alpha_{y} \gamma_{y} \alpha_{1} \\
& +\gamma_{x y}^{2} \gamma_{x x}^{2} \alpha_{x y}^{2} \gamma_{y y} \alpha_{y y}^{2} \alpha_{y}^{2} \gamma_{1}-4 \gamma_{y y}^{2} \gamma_{x x} \alpha_{x y}^{2} \gamma_{x y}^{2} \alpha_{x x} \alpha_{y} \gamma_{y} \alpha_{y y} \alpha_{1}-2 \gamma_{y y} \gamma_{x x}^{2} \alpha_{x y}^{3}
\end{aligned}
$$


$\gamma_{x y} \alpha_{y y}^{2} \gamma_{y}^{2} \alpha_{1}+2 \gamma_{y y}^{2} \alpha_{x y} \gamma_{x y}^{3} \alpha_{x x}^{2} \alpha_{y} \gamma_{y} \alpha_{y y} \alpha_{1}+2 \gamma_{y y} \gamma_{x x}^{2} \alpha_{x y}^{3} \gamma_{x y} \alpha_{y y}^{2} \gamma_{y} \alpha_{y} \gamma_{1}$ $+4 \gamma_{y y} \gamma_{x x} \alpha_{x y}^{2} \gamma_{x y}^{2} \alpha_{y y}^{2} \gamma_{y}^{2} \alpha_{x x} \alpha_{1}+\gamma_{y y}^{3} \gamma_{x x}^{2} \alpha_{x y}^{4} \alpha_{y}^{2} \gamma_{1}-4 \gamma_{y y} \gamma_{x x} \alpha_{x y}^{2} \gamma_{x y}^{2} \alpha_{y y}^{2} \gamma_{y}$ $\alpha_{x x} \alpha_{y} \gamma_{1}-\gamma_{y y}^{3} \gamma_{x x}^{2} \alpha_{x y}^{4} \alpha_{y} \gamma_{y} \alpha_{1}+2 \gamma_{y y}^{3} \gamma_{x x} \alpha_{x y}^{3} \alpha_{y} \gamma_{y} \alpha_{x x} \gamma_{x y} \alpha_{1}-2 \gamma_{y y}^{3} \gamma_{x x} \alpha_{x y}^{3}$ $\alpha_{y}^{2} \gamma_{1} \alpha_{x x} \gamma_{x y}-\gamma_{y y}^{3} \alpha_{x y}^{2} \alpha_{x x}^{2} \gamma_{x y}^{2} \alpha_{y} \gamma_{y} \alpha_{1}+\gamma_{y y}^{3} \alpha_{x y}^{2} \alpha_{x x}^{2} \gamma_{x y}^{2} \alpha_{y}^{2} \gamma_{1}+\gamma_{x y}^{2} \gamma_{x x}^{2} \alpha_{x y}^{2}$ $\alpha_{y y}^{3} \gamma_{y}^{2} \alpha_{1}-\gamma_{y y}^{2} \alpha_{x y}^{2} \alpha_{y y} \gamma_{y} \alpha_{x x}^{2} \gamma_{x y}^{2} \alpha_{y} \gamma_{1}-2 \gamma_{y y}^{2} \gamma_{x x} \alpha_{x y}^{3} \alpha_{x x} \gamma_{x y} \alpha_{y y} \gamma_{y}^{2} \alpha_{1}+\gamma_{y y}^{2}$ $\gamma_{x x}^{2} \alpha_{x y}^{4} \alpha_{y y} \gamma_{y}^{2} \alpha_{1}-\gamma_{x y}^{4} \alpha_{x x}^{2} \alpha_{y y}^{3} \gamma_{y} \alpha_{y} \gamma_{1}+4 \gamma_{y y}^{2} \gamma_{x x} \alpha_{x y}^{2} \gamma_{x y}^{2} \alpha_{x x} \alpha_{y}^{2} \gamma_{1} \alpha_{y y}+\gamma_{x y}^{4}$ $\alpha_{x x}^{2} \alpha_{y y}^{3} \gamma_{y}^{2} \alpha_{1}+2 \gamma_{x y}^{3} \alpha_{x x} \gamma_{x x} \alpha_{x y} \gamma_{y y} \alpha_{y y}^{2} \alpha_{y} \gamma_{y} \alpha_{1}-2 \gamma_{x y}^{3} \alpha_{x x} \gamma_{x x} \alpha_{x y} \gamma_{y y} \alpha_{y y}^{2} \alpha_{y}^{2}$ $\gamma_{1}-2 \gamma_{x y}^{3} \gamma_{x x} \alpha_{x y} \alpha_{x x} \alpha_{y y}^{3} \gamma_{y}^{2} \alpha_{1}+2 \gamma_{x y}^{3} \gamma_{x x} \alpha_{x y} \alpha_{x x} \alpha_{y y}^{3} \gamma_{y} \alpha_{y} \gamma_{1}+2 \gamma_{y y} \alpha_{x y} \gamma_{x y}^{3}$ $\alpha_{x x}^{2} \alpha_{y y}^{2} \gamma_{y} \alpha_{y} \gamma_{1}$

$3 \gamma_{x y} \gamma_{x x}^{2} \alpha_{x y}^{4} \gamma_{y}^{2} \alpha_{y}^{2} \gamma_{1}-3 \gamma_{x y} \gamma_{x x}^{2} \alpha_{x y}^{4} \gamma_{y}^{3} \alpha_{y} \alpha_{1}-\gamma_{x x}^{2} \alpha_{x y}^{5} \gamma_{y}^{3} \alpha_{y} \gamma_{1}+3 \gamma_{x y}^{3} \alpha_{x x}^{2} \gamma_{y}^{2}$ $\alpha_{x y}^{2} \alpha_{y}^{2} \gamma_{1}-\gamma_{x y}^{5} \alpha_{x x}^{2} \alpha_{y}^{3} \gamma_{y} \alpha_{1}+\gamma_{x y}^{3} \gamma_{x x}^{2} \alpha_{x y}^{2} \alpha_{y}^{4} \gamma_{1}+6 \gamma_{x y}^{3} \alpha_{x x} \gamma_{y} \gamma_{x x} \alpha_{x y}^{2} \alpha_{y}^{3} \gamma_{1}$

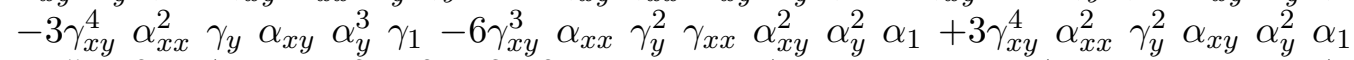
$+\gamma_{x y}^{5} \alpha_{x x}^{2} \alpha_{y}^{4} \gamma_{1}-3 \gamma_{x y}^{3} \alpha_{x x}^{2} \gamma_{y}^{3} \alpha_{x y}^{2} \alpha_{y} \alpha_{1}-2 \gamma_{x y}^{4} \gamma_{x x} \alpha_{x y} \alpha_{x x} \alpha_{y}^{4} \gamma_{1}+2 \gamma_{x y} \gamma_{x x} \alpha_{x y}^{4}$ $\gamma_{y}^{3} \alpha_{x x} \alpha_{y} \gamma_{1}-\gamma_{x y}^{2} \alpha_{x x}^{2} \gamma_{y}^{3} \alpha_{x y}^{3} \alpha_{y} \gamma_{1}-2 \gamma_{x y} \gamma_{x x} \alpha_{x y}^{4} \gamma_{y}^{4} \alpha_{x x} \alpha_{1}+2 \gamma_{x y}^{4} \gamma_{x x} \alpha_{x y} \alpha_{x x}$ $\alpha_{y}^{3} \gamma_{y} \alpha_{1}-\gamma_{x y}^{3} \gamma_{x x}^{2} \alpha_{x y}^{2} \alpha_{y}^{3} \gamma_{y} \alpha_{1}+\gamma_{x x}^{2} \alpha_{x y}^{5} \gamma_{y}^{4} \alpha_{1}+3 \gamma_{x y}^{2} \gamma_{x x}^{2} \alpha_{x y}^{3} \gamma_{y}^{2} \alpha_{y}^{2} \alpha_{1}-6 \gamma_{x y}^{2}$ $\alpha_{x x} \gamma_{y}^{2} \gamma_{x x} \alpha_{x y}^{3} \alpha_{y}^{2} \gamma_{1}+6 \gamma_{x y}^{2} \alpha_{x x} \gamma_{y}^{3} \gamma_{x x} \alpha_{x y}^{3} \alpha_{y} \alpha_{1}-3 \gamma_{x y}^{2} \gamma_{x x}^{2} \alpha_{x y}^{3} \gamma_{y} \alpha_{y}^{3} \gamma_{1}+\gamma_{x y}^{2}$ $\alpha_{x x}^{2} \gamma_{y}^{4} \alpha_{x y}^{3} \alpha_{1}$,

$\gamma_{x y}^{3} \alpha_{x x}^{2} \gamma_{x}^{2} \alpha_{x} \alpha_{y}^{3} \gamma_{1}+\gamma_{x} \gamma_{x x}^{2} \alpha_{x y}^{3} \alpha_{x}^{2} \gamma_{y}^{3} \alpha_{1}+\gamma_{x}^{3} \gamma_{x x}^{2} \alpha_{x y}^{3} \alpha_{y}^{2} \gamma_{y} \alpha_{1}+\gamma_{x y}^{3} \alpha_{x x}^{2} \alpha_{x}^{3}$ $\gamma_{y}^{2} \alpha_{y} \gamma_{1}-\gamma_{x}^{3} \alpha_{x y} \alpha_{x x}^{2} \gamma_{x y}^{2} \alpha_{y}^{3} \gamma_{1}+2 \gamma_{x y} \gamma_{x x}^{2} \alpha_{x y}^{2} \gamma_{x} \alpha_{x}^{2} \gamma_{y}^{2} \alpha_{y} \alpha_{1}+2 \gamma_{x y}^{2} \alpha_{x x} \gamma_{x x} \alpha_{x}^{3}$ $\gamma_{y}^{3} \alpha_{x y} \alpha_{1}+4 \gamma_{x y}^{2} \alpha_{x x} \gamma_{x} \gamma_{x x} \alpha_{x}^{2} \gamma_{y} \alpha_{x y} \alpha_{y}^{2} \gamma_{1}-4 \gamma_{x y}^{2} \alpha_{x x} \gamma_{x} \gamma_{x x} \alpha_{x}^{2} \gamma_{y}^{2} \alpha_{x y} \alpha_{y} \alpha_{1}$ $-2 \gamma_{x y}^{3} \alpha_{x x}^{2} \gamma_{x} \alpha_{x}^{2} \alpha_{y}^{2} \gamma_{y} \gamma_{1}+2 \gamma_{x y}^{3} \alpha_{x x}^{2} \gamma_{x} \alpha_{x}^{2} \alpha_{y} \gamma_{y}^{2} \alpha_{1}-\gamma_{x y}^{3} \alpha_{x x}^{2} \gamma_{x}^{2} \alpha_{x} \alpha_{y}^{2} \gamma_{y} \alpha_{1}$ $-\gamma_{x} \gamma_{x x}^{2} \alpha_{x y}^{3} \alpha_{x}^{2} \gamma_{y}^{2} \alpha_{y} \gamma_{1}+\gamma_{x y} \gamma_{x x}^{2} \alpha_{x y}^{2} \gamma_{x}^{2} \alpha_{x} \alpha_{y}^{3} \gamma_{1}-\gamma_{x y} \gamma_{x x}^{2} \alpha_{x y}^{2} \gamma_{x}^{2} \alpha_{x} \alpha_{y}^{2} \gamma_{y} \alpha_{1}$ $+2 \gamma_{x}^{2} \gamma_{x x}^{2} \alpha_{x y}^{3} \gamma_{y} \alpha_{y}^{2} \gamma_{1} \alpha_{x}-2 \gamma_{x}^{2} \gamma_{x x}^{2} \alpha_{x y}^{3} \gamma_{y}^{2} \alpha_{y} \alpha_{x} \alpha_{1}+2 \gamma_{x}^{3} \gamma_{x x} \alpha_{x y}^{2} \gamma_{x y} \alpha_{y}^{3} \gamma_{1} \alpha_{x x}$ $-2 \gamma_{x}^{3} \gamma_{x x} \alpha_{x y}^{2} \gamma_{x y} \alpha_{y}^{2} \gamma_{y} \alpha_{x x} \alpha_{1}+\gamma_{x y} \gamma_{x x}^{2} \alpha_{x y}^{2} \alpha_{x}^{3} \gamma_{y}^{2} \alpha_{y} \gamma_{1}+\gamma_{x}^{3} \alpha_{x y} \alpha_{x x}^{2} \gamma_{x y}^{2} \alpha_{y}^{2} \gamma_{y}$ $\alpha_{1}+2 \gamma_{x} \gamma_{x x} \alpha_{x y}^{2} \gamma_{x y} \alpha_{x}^{2} \gamma_{y}^{2} \alpha_{x x} \alpha_{y} \gamma_{1}-2 \gamma_{x} \gamma_{x x} \alpha_{x y}^{2} \gamma_{x y} \alpha_{x}^{2} \gamma_{y}^{3} \alpha_{x x} \alpha_{1}-4 \gamma_{x}^{2} \gamma_{x x}$ $\alpha_{x y}^{2} \gamma_{x y} \alpha_{x} \gamma_{y} \alpha_{y}^{2} \gamma_{1} \alpha_{x x}+4 \gamma_{x}^{2} \gamma_{x x} \alpha_{x y}^{2} \gamma_{x y} \alpha_{x} \gamma_{y}^{2} \alpha_{y} \alpha_{x x} \alpha_{1}+2 \gamma_{x}^{2} \alpha_{x y} \gamma_{x y}^{2} \alpha_{x} \alpha_{y}^{2}$ $\alpha_{x x}^{2} \gamma_{y} \gamma_{1}-2 \gamma_{x}^{2} \alpha_{x y} \gamma_{x y}^{2} \alpha_{x} \alpha_{y} \alpha_{x x}^{2} \gamma_{y}^{2} \alpha_{1}-2 \gamma_{x y}^{2} \alpha_{x x} \gamma_{x}^{2} \gamma_{x x} \alpha_{x} \alpha_{x y} \alpha_{y}^{3} \gamma_{1}+2 \gamma_{x y}^{2}$ $\alpha_{x x} \gamma_{x}^{2} \gamma_{x x} \alpha_{x} \alpha_{x y} \alpha_{y}^{2} \gamma_{y} \alpha_{1}-\gamma_{x}^{3} \gamma_{x x}^{2} \alpha_{x y}^{3} \alpha_{y}^{3} \gamma_{1}-\gamma_{x y}^{3} \alpha_{x x}^{2} \alpha_{x}^{3} \gamma_{y}^{3} \alpha_{1}-2 \gamma_{x y}^{2} \alpha_{x x} \gamma_{x x}$ $\alpha_{x}^{3} \gamma_{y}^{2} \alpha_{y} \gamma_{1} \alpha_{x y}-\gamma_{x y} \gamma_{x x}^{2} \alpha_{x y}^{2} \alpha_{x}^{3} \gamma_{y}^{3} \alpha_{1}-2 \gamma_{x y} \gamma_{x x}^{2} \alpha_{x y}^{2} \gamma_{x} \alpha_{x}^{2} \gamma_{y} \alpha_{y}^{2} \gamma_{1}-\gamma_{x} \alpha_{x x}^{2}$ $\gamma_{x y}^{2} \alpha_{x}^{2} \gamma_{y}^{2} \alpha_{x y} \alpha_{y} \gamma_{1}+\gamma_{x} \alpha_{x x}^{2} \gamma_{x y}^{2} \alpha_{x}^{2} \gamma_{y}^{3} \alpha_{x y} \alpha_{1}$,

$6 \gamma_{x x}^{2} \alpha_{x x} \gamma_{x}^{2} \alpha_{x}^{3} \gamma_{y} \alpha_{y}^{2} \gamma_{1}-\gamma_{x x}^{3} \alpha_{x}^{3} \gamma_{x}^{2} \alpha_{y}^{2} \gamma_{y} \alpha_{1}-\gamma_{x x}^{3} \alpha_{x}^{5} \gamma_{y}^{3} \alpha_{1}-6 \gamma_{x x}^{2} \alpha_{x x} \gamma_{x}^{2} \alpha_{x}^{3}$ $\gamma_{y}^{2} \alpha_{y} \alpha_{1}+6 \gamma_{x x} \alpha_{x x}^{2} \gamma_{x}^{3} \gamma_{y}^{2} \alpha_{x}^{2} \alpha_{y} \alpha_{1}-\alpha_{x x}^{3} \gamma_{x}^{5} \alpha_{y}^{3} \gamma_{1}+\gamma_{x x}^{3} \alpha_{x}^{5} \gamma_{y}^{2} \alpha_{y} \gamma_{1}+3 \gamma_{x x}^{2} \alpha_{x x}$ $\gamma_{x}^{3} \alpha_{x}^{2} \alpha_{y}^{2} \gamma_{y} \alpha_{1}+\gamma_{x x}^{3} \alpha_{x}^{3} \gamma_{x}^{2} \alpha_{y}^{3} \gamma_{1}+\alpha_{x x}^{3} \gamma_{x}^{5} \alpha_{y}^{2} \gamma_{y} \alpha_{1}-\alpha_{x x}^{3} \gamma_{x}^{3} \alpha_{x}^{2} \gamma_{y}^{2} \alpha_{y} \gamma_{1}+3 \gamma_{x x}^{2}$

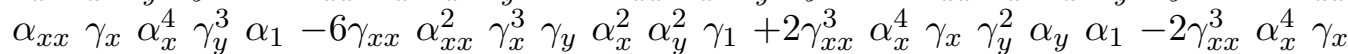
$\gamma_{y} \alpha_{y}^{2} \gamma_{1}+\alpha_{x x}^{3} \gamma_{x}^{3} \alpha_{x}^{2} \gamma_{y}^{3} \alpha_{1}-3 \gamma_{x x}^{2} \alpha_{x x} \gamma_{x}^{3} \alpha_{x}^{2} \alpha_{y}^{3} \gamma_{1}+3 \gamma_{x x} \alpha_{x x}^{2} \gamma_{x}^{4} \alpha_{x} \alpha_{y}^{3} \gamma_{1}-3 \gamma_{x x}$ $\alpha_{x x}^{2} \gamma_{x}^{4} \alpha_{x} \alpha_{y}^{2} \gamma_{y} \alpha_{1}-3 \gamma_{x x}^{2} \alpha_{x x}, \gamma_{x} \alpha_{x}^{4} \gamma_{y}^{2} \alpha_{y} \gamma_{1}-3 \gamma_{x x} \alpha_{x x}^{2} \gamma_{x}^{2} \alpha_{x}^{3} \gamma_{y}^{3} \alpha_{1}-2 \alpha_{x x}^{3} \gamma_{x}^{4}$ $\alpha_{x} \gamma_{y}^{2} \alpha_{y} \alpha_{1}+2 \alpha_{x x}^{3} \gamma_{x}^{4} \alpha_{x} \gamma_{y} \alpha_{y}^{2} \gamma_{1}+3 \gamma_{x x} \alpha_{x x}^{2} \gamma_{x}^{2} \alpha_{x}^{3} \gamma_{y}^{2} \alpha_{y} \gamma_{1}$,

$3 \alpha_{x x}^{3} \gamma_{x}^{4} \alpha_{x}^{2} \alpha_{1} \gamma_{1}^{2}+3 \gamma_{x x}^{3} \alpha_{x}^{4} \gamma_{x}^{2} \gamma_{1} \alpha_{1}^{2}+\gamma_{x x}^{3} \alpha_{x}^{6} \gamma_{1}^{3}+\alpha_{x x}^{3} \gamma_{x}^{6} \alpha_{1}^{3}-3 \gamma_{x x}^{3} \alpha_{x}^{5} \gamma_{x} \gamma_{1}^{2} \alpha_{1}$ $+9 \gamma_{x x} \alpha_{x x}^{2} \gamma_{x}^{4} \alpha_{x}^{2} \alpha_{1}^{2} \gamma_{1}+3 \gamma_{x x} \alpha_{x x}^{2} \gamma_{x}^{2} \alpha_{x}^{4} \gamma_{1}^{3}+3 \gamma_{x x}^{2} \alpha_{x x} \gamma_{x}^{4} \alpha_{x}^{2} \alpha_{1}^{3}-3 \alpha_{x x}^{3} \gamma_{x}^{5} \alpha_{x} \alpha_{1}^{2}$ $\gamma_{1}-9 \gamma_{x x}^{2} \alpha_{x x} \gamma_{x}^{3} \alpha_{x}^{3} \gamma_{1} \alpha_{1}^{2}-3 \gamma_{x x}^{2} \alpha_{x x} \gamma_{x} \alpha_{x}^{5} \gamma_{1}^{3}-3 \gamma_{x x} \alpha_{x x}^{2} \gamma_{x}^{5} \alpha_{x} \alpha_{1}^{3}+9 \gamma_{x x}^{2} \alpha_{x x} \gamma_{x}^{2}$ $\alpha_{x}^{4} \gamma_{1}^{2} \alpha_{1}-\alpha_{x x}^{3} \gamma_{x}^{3} \alpha_{x}^{3} \gamma_{1}^{3}-\gamma_{x x}^{3} \alpha_{x}^{3} \gamma_{x}^{3} \alpha_{1}^{3}-9 \gamma_{x x} \alpha_{x x}^{2} \gamma_{x}^{3} \alpha_{x}^{3} \gamma_{1}^{2} \alpha_{1}$ 


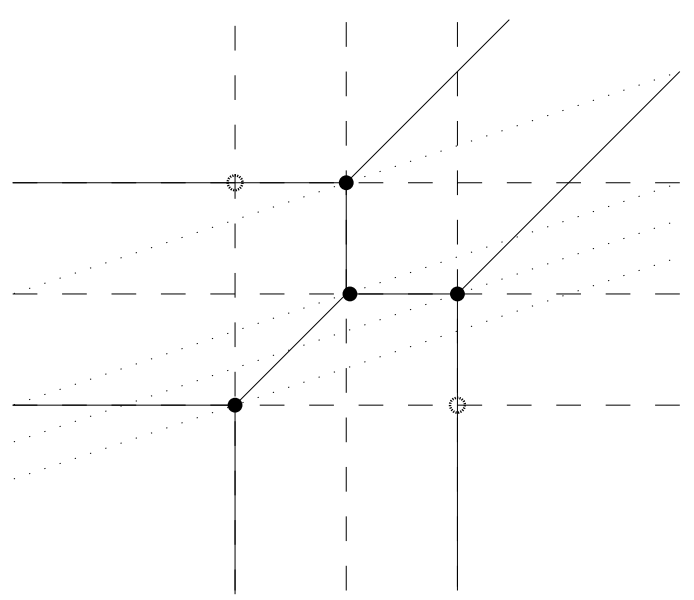

Figure 1: Three resultants are needed to compute the stable intersection.

\section{Some remarks}

As a consequence of Theorem 17, a new proof of Bernstein-Koushnirenko Theorem for plane curves over an arbitrary algebraically closed field can be derived from the classic Theorem over $\mathbb{C}([1]$, [7]). We refer to [10] for a direct proof in positive characteristic.

Corollary 20. Let $\tilde{f}, \widetilde{g}$ be two polynomials over $\mathbb{K}$, an algebraically closed. Let $\Delta_{f}, \Delta_{g}$ be the Newton polytope of the polynomials $\widetilde{f}$ and $\widetilde{g}$ respectively. Then, if the coefficients of $\widetilde{f}$ and $\widetilde{g}$ are generic, then the number of common roots of the curves in $\left(\mathbb{K}^{*}\right)^{2}$ counted with multiplicities is the mixed volume of the Newton polygons

$$
\mathcal{M}\left(\Delta_{f}, \Delta_{g}\right)=\operatorname{vol}\left(\Delta_{f}+\Delta_{g}\right)-\operatorname{vol}\left(\Delta_{f}\right)-\operatorname{vol}\left(\Delta_{g}\right)
$$

Proof. If the coefficients of the polynomials are generic, the number of roots in the torus counted with multiplicities is the degree minus the order of the resultant of the two polynomials with respect to one of the variables. This number does only depend on the support of the polynomials, and it is equal to the mixed volume of the Newton polygons, because this is the number of stable intersection points of two tropical curves of Newton polygons $\Delta_{f}, \Delta_{g}$.

Remark 21. Another application of the techniques developed in this article is the computation of tropical bases. Theorem 1 proves that for a hypersurface $\widetilde{f}$, the projection $T(\{\widetilde{f}=0\})=\mathcal{T}(f)$. This is not true for general ideals. If $\mathcal{I}=\left(\widetilde{f}_{1}, \ldots, \widetilde{f}_{m}\right) \subseteq \mathbb{K}\left[x_{1}, \ldots, x_{n}\right]$ and $\mathcal{V}$ is the variety it defines 
in $\left(\mathbb{K}^{*}\right)^{n}$,

$$
T(\mathcal{V}) \subseteq \bigcap_{i=1}^{m} \mathcal{T}\left(f_{i}\right),
$$

but it is possible that both sets are different. A set of generators $\widetilde{g}_{1}, \ldots, \widetilde{g}_{r}$ of $\mathcal{I}$ such that $T(\mathcal{V})=\bigcap_{i=1}^{r} \mathcal{T}\left(g_{r}\right)$ is called a tropical basis of $\mathcal{I}$. In [2], it is proved that every ideal has a tropical basis and it is provided an algorithm for the case of a prime ideal $\mathcal{I}$.

An alternative for the computation of a tropical basis of a zero dimensional ideal in two variables is the following. Let $\mathcal{I}=(\widetilde{f}, \widetilde{g})$ be a zero dimensional ideal in two variables. Let $\widetilde{R}_{x}, \widetilde{R}_{y}$ be the resultants with respect to $x$ and $y$ of the curves. Let $P$ be the intersection of the projections $R_{x}$ and $R_{y}$. This is always a finite set that contains the projection of the intersection of $\widetilde{f}, \widetilde{g}$. It may happen that $P$ is not contained in the stable intersection of the corresponding tropical curves $f$ and $g$, though. Let $a$ be a natural number such that $x-a y$ is injective in $P$. Let $\widetilde{R}_{z}=\operatorname{Res}_{y}\left(\widetilde{f}\left(z y^{a}, y\right), \widetilde{g}\left(z y^{a}, y\right)\right)$ be another resultant. Then, it follows that $\left(\widetilde{f}, \widetilde{g}, \widetilde{R}_{x}, \widetilde{R}_{y}, \widetilde{R}_{z}\right)$ is a tropical basis of the ideal $(\tilde{f}, \widetilde{g})$. This alternative approach is very similar to the regular projection method that has been developed by Hept and Theobald [5].

Remark 22. Along the article, the notion of tropical resultant has been defined as the projection of the algebraic resultant. It is needed a precomputation of the algebraic resultant in order to tropicalize it. For the case of plane curves, it would be preferable to have a determinantal formula. That is, to prove that the determinant of the Sylvester matrix of two polynomials define the resultant variety. But the proof of the properties is achieved by a careful look to the polynomials involved, paying special attention to the cancellation of terms. In the case of the determinant of the Sylvester matrix, the tropical determinant of the Sylvester matrix is the projection of the permanent of the algebraic determinant. There are cancellation of terms even in the equicharacteristic zero case. It is conjectured that still the determinant of the Sylvester matrix is a tropical polynomial that defines the same tropical variety as the resultant does. The author has checked that it is the case for polynomials up to degree four with full support.

\section{References}

[1] Bernstein, D. N.: The number of roots of a system of equations. Funkcional Anal. i Priložen 9 (1975), no. 3, 1-4.

[2] Bogart, T., Jensen, A. N., Speyer, D., Sturmfels, B. And Thomas, R. R.: Computing tropical varieties. J. Symbolic Comput. 42 (2007), no. $1-2,54-73$. 
[3] Einsiedler, M., Kapranov, M. And Lind, D.: Non-Archimedean amoebas and tropical varieties. J. Reine Angew. Math. 601 (2006), 139-157.

[4] Gel'fand, I. M., Kapranov, M. M. and Zelevinsky, A. V.: Newton polytopes of the classical resultant and discriminant. Adv. Math. 84 (1990), no. $2,237-254$.

[5] Hept, K. And Theobald, T.: Tropical bases by regular projections. Preprint, 2007. Electronic version at http://arxiv.org/abs/0708.1727.

[6] Jensen, A. N., Markwig, H. And Markwig, T.: An algorithm for lifting points in a tropical variety. Collect. Math. 59 (2008), no. 2, 129-165.

[7] Kushnirenko, A. G.: Newton polyhedra and Bezout's theorem. Funkcional Anal. i Priložen 10 (1976), no. 3, 82-83.

[8] Mikhalkin, G.: Enumerative tropical algebraic geometry in $\mathbb{R}^{2}$. J. Amer. Math. Soc. 18 (2005), no. 2, 313-377 (electronic).

[9] Richter-Gebert, J., Sturmfels, B. and Theobald, T.: First steps in tropical geometry. In Contemp. Math. 377, 289-317. Amer. Math. Soc., Providence, RI, 2005.

[10] Rojas, J. M.: Toric intersection theory for affine root counting. J. Pure Appl. Algebra 136 (1999), no. 1, 67-100.

[11] Sturmfels, B.: On the Newton polytope of the resultant. J. Algebraic Combin. 3 (1994), no. 2, 207-236.

[12] Sturmfels, B.: Solving systems of polynomial equations. In CBMS Regional Conference Series in Mathematics 97. Conference Board of the Mathematical Sciences, Washington, DC, 2002. Amer. Math. Soc., Providence, RI, 2002.

[13] Tabera, L. F.: Tropical constructive Pappus' theorem. Int. Math. Res. Not. 39 (2005), 2373-2389.

[14] TABera, L. F.: Constructive proof of extended Kapranov theorem. In Actas del X Encuentro de Álgebra Computacional y Aplicaciones, 178-181. EACA, 2006.

Recibido: 4 de junio de 2007

Luis Felipe Tabera

IMDEA Matemáticas Facultad de Ciencias C-IX Campus Universidad Autónoma de Madrid E-28049 Madrid, Spain. luis.tabera@imdea.org

The author is supported by the project MTM2005-08690-C02-02 and a FPU research grant from the Spanish Ministerio de Educación y Ciencia. 